\title{
Dose profiles for lung and breast regions at prospective and retrospective CT coronary angiography using optically stimulated luminescence dosimeters on a 64-detector CT scanner
}

\section{Yoshinori Funama $^{\mathrm{a}, *}$, Katsuyuki Taguchi ${ }^{\mathrm{b}}$, Daisuke Utsunomiya ${ }^{\mathrm{c}}$, Seitaro Oda c, Hiroo Murasaki ${ }^{d}$, Yasuyuki Yamashita ${ }^{d}$, Kazuo Awai ${ }^{\text {e }}$}

a Department of Medical Physics, Faculty of Life Sciences, Kumamoto University, 4-24-1 Kuhonji, Kumamoto 862-0976, Japan

b The Russell H. Morgan Department of Radiology and Radiological Science, The Johns Hopkins University School of Medicine, Baltimore, Maryland, United States

' Department of Diagnostic Radiology, Graduate School of Medical Sciences, Kumamoto University, Kumamoto, Japan

${ }^{\mathrm{d}}$ Course of Radiological Sciences, Graduate School of Health Sciences, Kumamoto University, Kumamoto, Japan

e Department of Diagnostic Radiology, Graduate School of Biomedical Sciences, Hiroshima University, Hiroshima, Japan

Received 12 July 2010; received in revised form 5 January 2011; accepted 22 January 2011

Available online 25 February 2011

\section{KEYWORDS}

OSL dosimeter;

Dose profile;

CTCA;

Prospective mode;

Retrospective mode

\begin{abstract}
Purpose: The purpose of our study was to acquire dose profiles at critical organs of lung and breast regions using optically stimulated luminescence (OSL) dosimeters; assess the actual radiation dose delivered at retrospective and prospective computed tomography coronary angiography (CTCA).

Materials and methods: Using a chest CT phantom we applied a prospectively-gated step-andshoot- and a retrospectively-gated helical mode on a 64-detector row CT scanner. Retrospective scan mode was used with and without electrocardiogram (ECG) based tube current modulation. OSL dosimeters were used to measure dose profiles. In the both scan modes we acquired dose profiles and determined the mean and maximum dose in left lung and in left breast regions. Results: In prospective mode, the mean dose was $21.53 \mathrm{mGy}$ in left lung- and $23.59 \mathrm{mGy}$ in left breast region. With respect to the retrospective mode, the mean dose with tube current modulation was $38.63 \mathrm{mG}$ for left lung- and $46.02 \mathrm{mGy}$ for left breast region, i.e. 0.56 and 0.55 times lower than the mean dose without modulation.
\end{abstract}

\footnotetext{
* Corresponding author. Tel.: +8196373 5455; fax: +81963735455.
}

E-mail address: funama@kumamoto-u.ac.jp (Y. Funama).

1120-1797/\$ - see front matter ( $) 2011$ Associazione Italiana di Fisica Medica. Published by Elsevier Ltd. All rights reserved. doi:10.1016/j.ejmp.2011.01.003 
Conclusion: The OSL dosimeter is useful for measurement of the actual radiation dose along $\mathrm{z}$-axis at lung and breast regions in the prospective and the retrospective CTCA.

(c) 2011 Associazione Italiana di Fisica Medica. Published by Elsevier Ltd. All rights reserved.

\section{Introduction}

Multidetector computed tomography coronary angiography (CTCA) is an accurate method for the non-invasive diagnosis of coronary artery disease [1-5]. CTCA acquisition protocols using helical and step-and-shoot scanning have been employed for use with retrospective and prospective electrocardiogram (ECG) triggering [6-11]. The major issue of the retrospective scan mode is that it entails a high radiation dose during CT scanning because only part of the total radiation delivered is used for the reconstruction of the image $[4,9]$. To overcome the radiation dose problem, prospective CTCA is employed. Prospective CTCA acquisition technique facilitates coverage of the whole heart in 3 or 4 steps and is different from retrospective scan mode $[9,12,13]$.

We focused on the measurement of actual radiation dose for lung and breast regions undergoing prospective and retrospective CTCA. To measure the radiation dose we use optically stimulated luminescence (OSL) dosimeters for CT dosimetry [14] This is an advantage to measure the actual radiation dose along the z-axis. Because the OSL dosimeter allows the delivery of radiation doses at $0.05 \mathrm{~mm}$ intervals in $150 \mathrm{~mm}$ lengths, it allows the acquisition of detailed dose information. Consequently, the OSL dosimeter can provide not only the dose value but also dose profiles.

In this study we use a chest CT phantom to acquire dose profiles at critical organs of lung and breast regions using OSL dosimeters; assess the actual radiation dose delivered at retrospective and prospective CTCA.

\section{Materials and methods}

\section{Optically stimulated luminescence (OSL) dosimeters}

To measure the radiation dose we used OSL dosimeters for CT dosimetry (Landauer, Glenwood, IL, USA) [14]. Unlike the CT pencil ionization chamber, the OSL dosimeter provides not only the cumulative value but also the dose profiles. The strips for CT dosimetry were rectangle of $175 \mathrm{~mm}$ long and $12 \mathrm{~mm}$ diameter and placed in a small plastic cylinder. The radiation dose for the dose profiles was comprised of values measured at $0.05 \mathrm{~mm}$ intervals. The OSL dosimeter is made of aluminum oxide doped with carbon $\left(\mathrm{Al}_{2} \mathrm{O}_{3}: \mathrm{C}\right)$ as the dosimeter material. This material is commonly used in personal dosimeters worn by workers. To read the signal, the area of interest is stimulated by light from a laser or a light-emitting diode (LED) array (at a wavelength of approximately $520 \mathrm{~nm}$ ). The luminescence (at approximately $420 \mathrm{~nm}$ ) stimulated by this process is measured with a photomultiplier tube featuring photoncounting circuitry. The OSL dosimeter has a resolution of $0.2 \mathrm{~mm}$. This is achieved by using optical slits to limit the area of the dosimeter that is stimulated and also the area from which the luminescence is collected. The OSL signal is converted to an OSL reading using a pre established calibration factor which is determined using OSL dosimeters irradiated with a known dose from a ${ }^{90} \mathrm{Sr} /{ }^{90} \mathrm{Y}$ beta source (calibrated in ${ }^{60} \mathrm{Co}$ gamma ray dose to water against a NIST standard) [15]. The OSL dosimeter is calibrated to using an $\mathrm{x}$-ray technique (120 kVp, $8.3 \mathrm{~mm}$ Al half value layer $(\mathrm{HVL})$ ) designed to simulate the beam quality of a typical CT scanner. The reference exposure is performed in air with a fixed position x-ray generator. This allows the dosimeter to be used in any position of the CT phantom with accurate results while simultaneously correcting for the energy dependence of the OSL material. The fading of the dosimeter is negligible $(<2 \%)$ as the OSL strip is fully contained in a light-tight case. The minimum measurable dose is 5 mRad. The transit dose is accounted for using a separate control dosimeter which is shipped from Landauer at the same time as the test dosimeter.

\section{Chest CT phantom}

We used a multi-purpose chest CT phantom (N1, Kyoto Kagaku Co., Kyoto, Japan) that simulates an adult male with a chest circumference of $94 \mathrm{~cm}$. Simulated soft tissues such as pulmonary vessels, the chest wall, heart, diaphragm, and liver consist of polyurethane resin composites, simulated bone of an epoxide resin. The space between the pulmonary vessels, heart, and chest wall is filled with air and the chest wall can be removed from the other structures such as the simulated heart, pulmonary vessels, diaphragm, and liver.

We inserted the OSL dosimeters into the left lung- and left breast (chest surface) regions of the chest CT phantom along the $\mathrm{z}$-axis to cover the scan field-of-view (FOV) (Fig. 1). In retrospective mode using helical scans, we aligned 2 OSL dosimeters along the z-axis to evaluate the radiation dose outside the image FOV (overrange scan dose) of each region $[16,17]$. After exposure, the OSL dosimeters were returned to the manufacturer for reading with a reader and the read-out values were reported to us. The energy correction factor was used at effective energy of $64 \mathrm{keV}$ (tube voltage: $120 \mathrm{kV}$ ).

\section{Step-and-shoot- and helical scanning}

Using a 64-detector row CT scanner (Brilliance-64, Philips Medical Systems, Cleveland, OH, USA) we applied 2 scan modes used in routine clinical practice, a prospectivelygated step-and-shoot- and a retrospectively-gated helical mode. During the acquisition of step-and-shoot scans the table bearing the patient remains stationary. On the other hand, during helical scanning the table moves continuously during data acquisition. The prospectively-gated step-andshoot method involves prospective electrocardiogram (ECG) triggering, consequently, irradiation is delivered only 


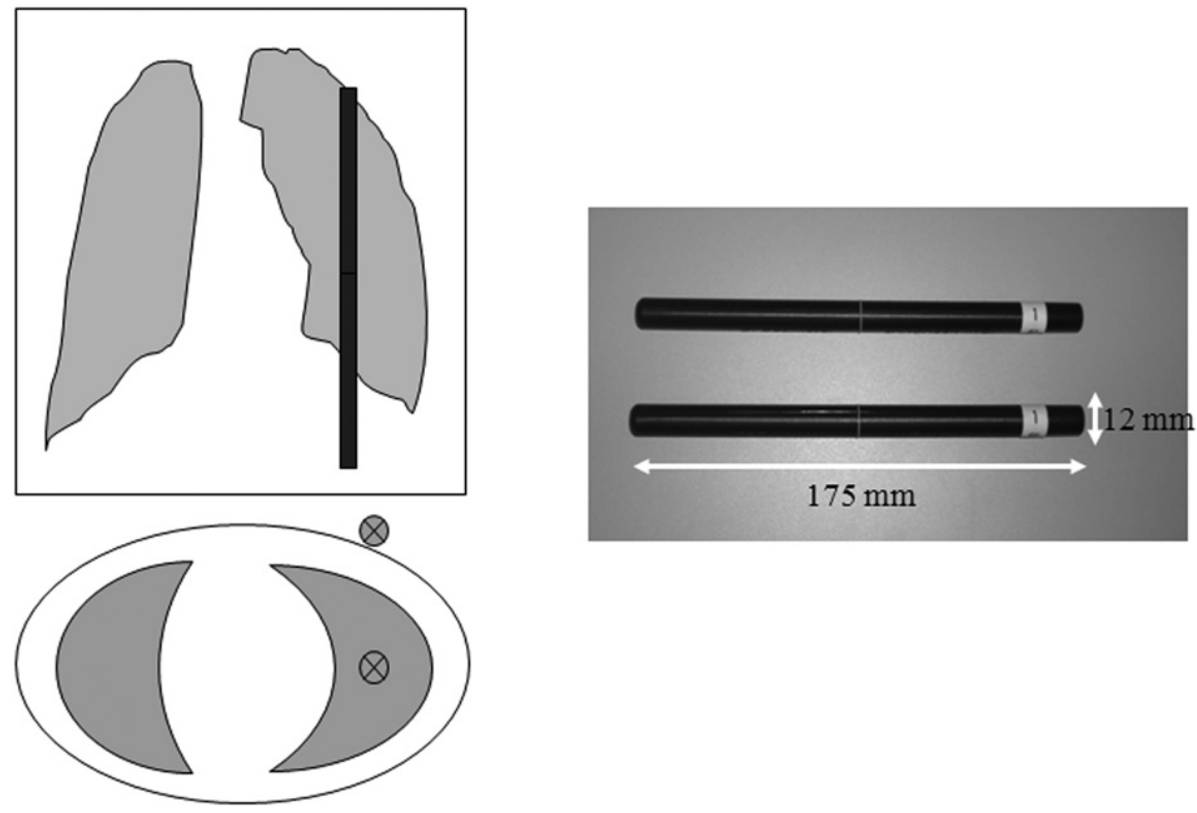

Figure 1 Optically stimulated luminescence (OSL) dosimeters placed in left lung- and left breast (chest surface) regions of the chest CT phantom along the z-axis to cover the scan field-of-view (FOV). In the retrospective mode using helical scans, 2 OSL dosimeters were placed along the $z$-axis to evaluate the radiation dose outside the image FOV (overrange scan dose) of each region. The strips for CT dosimetry were placed in a small (175 mm long, $12 \mathrm{~mm}$ diameter) plastic cylinder. The radiation dose profiles are measured in $0.05 \mathrm{~mm}$ intervals.

at predefined time points during the cardiac cycle. The parameters for the step-and-shoot scans were: detector configuration $64 \times 0.625 \mathrm{~mm}$ (detector collimation), slice thickness $0.8 \mathrm{~mm}$, gantry rotation time $0.42 \mathrm{~s}$. Tube voltage and tube current-time product were $120 \mathrm{kV}$ and $210 \mathrm{mAs}$, respectively. The ECG measurement was performed during continuous CT data acquisition using the demo mode at a heart rate of 60 beats per minute (bpm). The center of the imaging window was set at $75 \%$ of the $R-R$ interval. When the table was stationary, prospective scan data were acquired with a 40-mm step-and-shoot scan. The distance covered by the moving table was $32 \mathrm{~mm}$, this allowed for an 8-mm overlap for the subsequent data acquisition step. We set the scan length in the z-axis at $124.8 \mathrm{~mm}$ (4 steps of the step-and-shoot scan plus 3 overlap areas). The weighted CT dose index (CTDIw) displayed on the operator console was 15.8 $\mathrm{mGy}$; the value can be generated using the formula CTDIw $=2 / 3 \times$ CTDIp $+1 / 3 \times$ CTDIc, where CTDIp and CTDIc are the measured values at the surface and center of the 32-cm diameter acrylic phantom [14].

Retrospectively-gated helical scanning is performed with retrospective electrocardiogram (ECG) triggering. Consequently, irradiation is delivered continuously, whereas image reconstruction is restricted to data acquired at predefined time points in the cardiac cycle. The scan parameters for the retrospective scan mode were detector
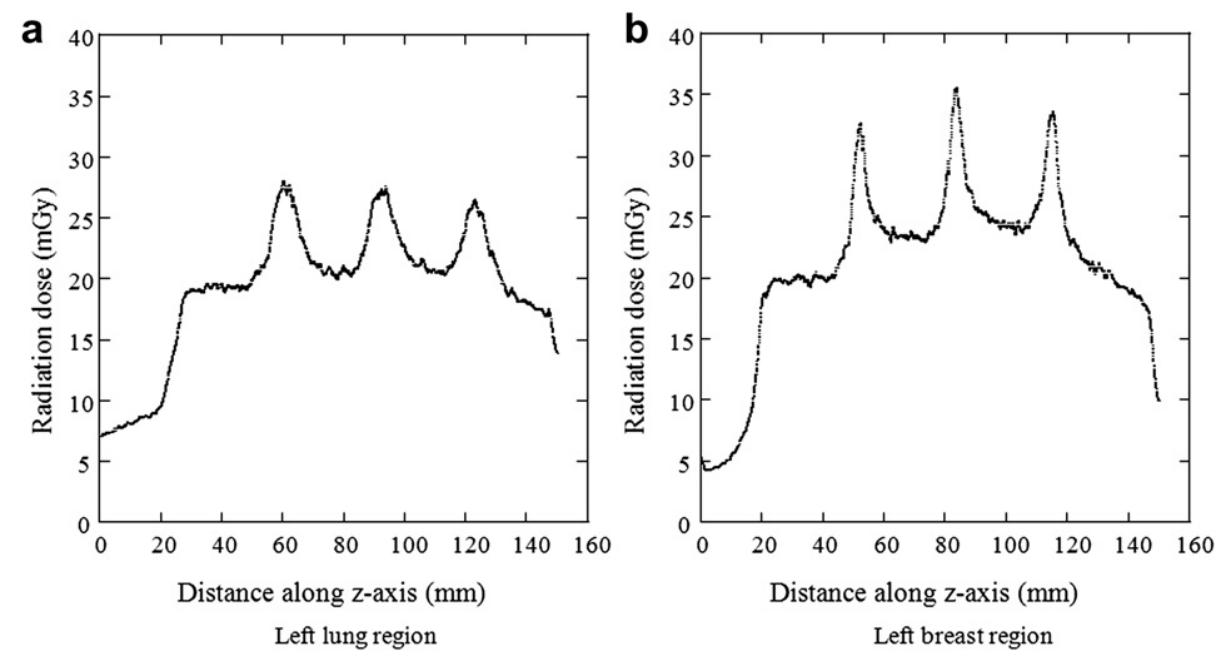

Figure 2 Dose profiles under prospective scan mode: (a) left lung region, (b) left breast region. 
Table 1 Mean and maximum scan doses with prospective CTCA.

\begin{tabular}{lll}
\hline & Left lung & Left breast \\
\hline Mean scan dose (mGy) & $21.53 \pm 2.03$ & $23.59 \pm 2.13$ \\
Maximum scan dose (mGy) & 28.04 & 35.52 \\
Accuracy (\%) & 9.42 & 9.02 \\
CTDIw (mGy) & 15.8 & \\
DLP (mGy cm) & 169.88 & \\
\hline
\end{tabular}

Mean and maximum scan doses were calculated from the value of scan profile curve within image scan length.

configuration $64 \times 0.625 \mathrm{~mm}$ (detector collimation), slice thickness $0.8 \mathrm{~mm}$, gantry rotation time $0.42 \mathrm{~s}$, beam pitch 0.2 , scan length (image scan length) $124.8 \mathrm{~mm}$. We defined the beam pitch as the distance traveled by the table in the course of one $360^{\circ}$ rotation/total collimated width of the $x$-ray beam [18]. ECG was performed during continuous CT data acquisition using the demo mode at a heart rate of $60 \mathrm{bpm}$. The effective current-time product, defined as the tube current-time product divided by the beam pitch, and the tube voltage were 900 effective mAs (corresponding to180 mAs) and $120 \mathrm{kV}$, respectively, with or without the ECG-based tube current modulation technique. The ECG-based tube current modulation technique applied to obtain radiation reduction involved a tube current-time product of $180 \mathrm{mAs}$ during $75 \% \pm 72 \mathrm{~ms}$ of the $\mathrm{R}-\mathrm{R}$ interval. The calculated volume CTDI (CTDIvol) displayed on the operator console was $52.9 \mathrm{mGy}$; the value is defined as CTDIw divided by the beam pitch [14].

\section{Data analysis}

We acquired dose profiles with the prospective and retrospective scan modes in the left lung and left breast regions. The mean and maximum radiation doses were determined from the dose profiles. In addition, percentage accuracy was calculated from mean value and standard deviation (SD) of the dose profile. Specifically, first we measured the radiation dose along the z-axis using OSL dosimeters. Then we obtained dose profiles for the left lung- and left breast regions from these measurements and calculated the mean/maximum radiation dose delivered to the left lung- and left breast region using the dose values from the dose profiles in both scan modes. We defined the maximum value within the image scan length as the maximum radiation dose. With the prospective scan mode, the maximum radiation dose corresponded to the radiation dose delivered at the point of overlapping. The mean values were calculated from the measurement values within the image scan length and excluded the measured values in the overrange area. In calculations of the overrange scan dose, the exposed scan length $(L)$ including the additional rotations at the beginning and end of the scan (overrange scan length) was calculated using the equation $[16,17]$ :

$$
\begin{array}{r}
L_{s}=n \times T F+b_{w}: \begin{array}{l}
\text { step }- \text { and }- \text { shoot scan } \\
\quad(\text { prospective scan mode })
\end{array} \\
L_{h}=T / t_{\text {rot }} \times \mathrm{p} \times b_{w}: \text { helical scan }
\end{array}
$$

(retrospective scan mode)

where $n \times T F$ is the number ( $n$ ) of table feeds (TF), $T$ is the total scan time, $t_{\text {rot }}$ the rotation time, $p$ the beam pitch factor, and $b_{w}$ the beam width. The exposed scan length for step-and-shoot scans $\left(L_{s}\right)$ was $136 \mathrm{~mm}$ and the exposed scan length for helical scans $\left(L_{h}\right)$ was $178.95 \mathrm{~mm}$. Therefore, the over scan length was $11.2 \mathrm{~mm}$ for step-and-shoot scans and $54.15 \mathrm{~mm}$ for helical scans. The exposed- and overrange scan doses were calculated from the integrated values within the exposed scan length and the overrange scan length, respectively. The radiation dose ratio $(R)$ between the overrange- and the exposed scan dose $\left(\right.$ dose $_{o}$, dose $\left._{e}\right)$ was calculated as:

$$
R=\text { dose }_{o} / \text { dose }_{e} \times 100(\%)
$$

For the prospectively-gated step-and-shoot- and the retrospectively-gated helical mode, image noise was measured at the center of the mediastinum region of the chest
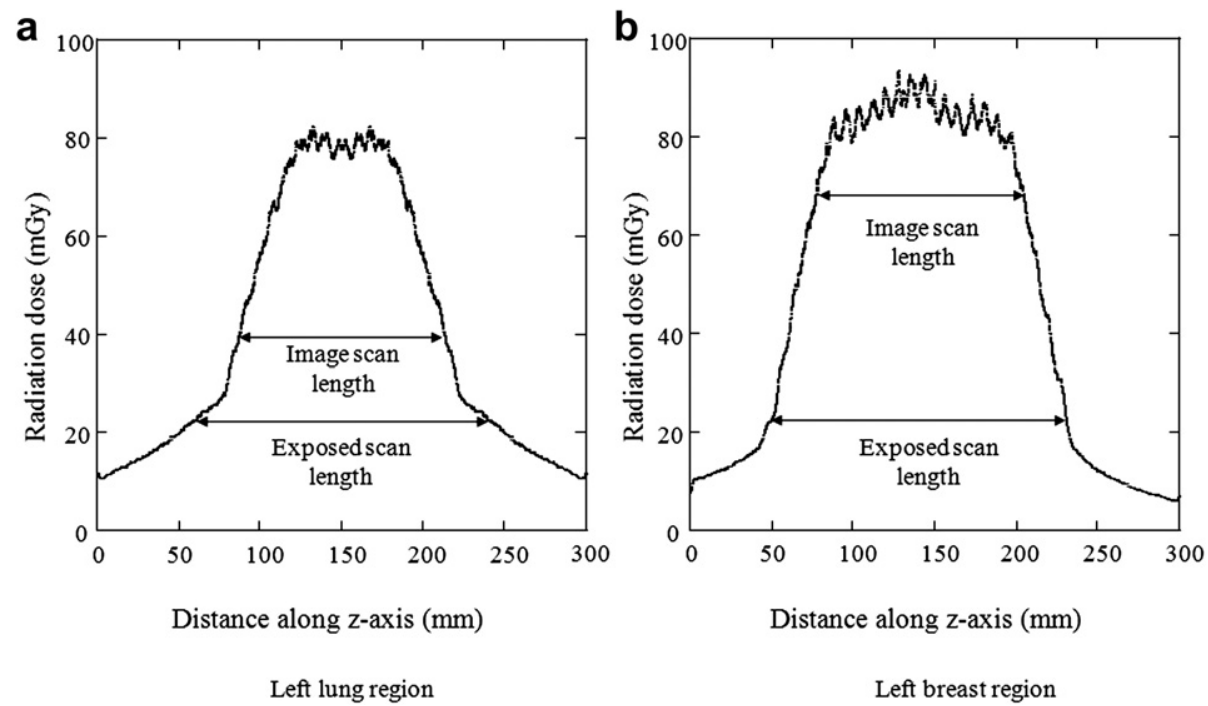

Figure 3 Dose profiles under retrospective scan mode without the ECG-based tube current modulation technique: (a) left lung region, (b) left breast region. 


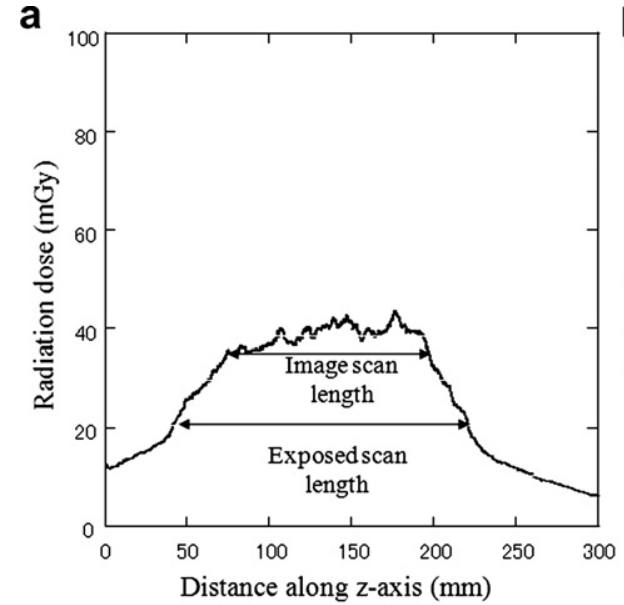

Left lung region

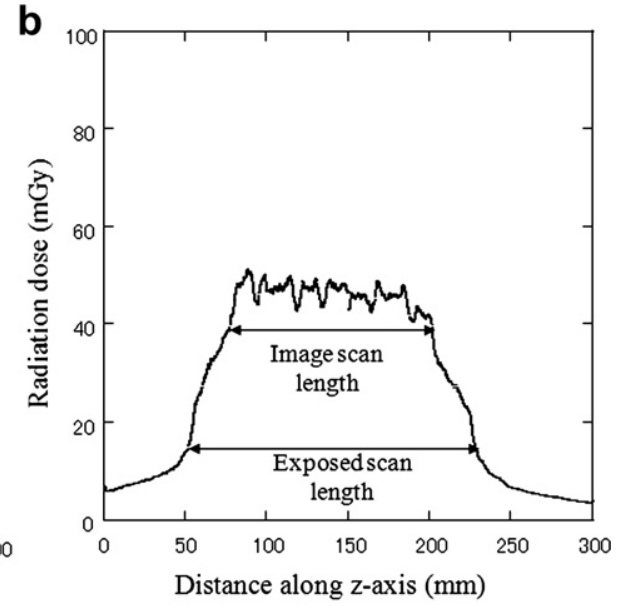

Left breast region

Figure 4 Dose profiles under retrospective scan mode with the ECG-based tube current modulation technique: (a) left lung region, (b) left breast region.

CT phantom; the noise index was the SD of the pixel values within a $50-\mathrm{mm}$ diameter circular region of interest (ROI).

\section{Results}

\section{Prospective CTCA}

With respect to the prospective scan mode, dose profiles in the left lung- and left breast regions (Fig. 2) revealed 3 overlap areas between the scans. The mean and SD measurement dose within the image scan length along the z-axis was $21.53 \pm 2.03 \mathrm{mGy}$ in the left lung- and $23.59 \pm 2.13 \mathrm{mGy}$ in the left breast region (Table 1 ). The maximum dose for overlap was $28.04 \mathrm{mGy}$ at left lung and $35.52 \mathrm{mGy}$ at left breast region (Table 1 ).

\section{Retrospective CTCA}

For comparison of the results obtained with and without the ECG-based tube current modulation technique, Figs. 3 and 4 show the dose profiles in the left lung- and left breast regions. The mean and SD dose without tube current modulation at helical scanning was $69.0 \pm 7.23 \mathrm{mGy}$ for the left lung and $83.7 \pm 8.12 \mathrm{mGy}$ for the left breast (Table $2 \mathrm{a}$ ).

Table 2a Mean and maximum scan doses with retrospective CTCA without ECG-based tube current modulation technique.

\begin{tabular}{lll}
\hline & Left lung & Left breast \\
\hline Mean scan dose (mGy) & $69.00 \pm 7.23$ & $83.70 \pm 8.12$ \\
Maximum scan dose (mGy) & 82.36 & 93.64 \\
Accuracy (\%) & 10.47 & 9.70 \\
CTDlvol (mGy) & 52.9 & \\
DLP (mGy cm) & 947.66 & \\
\hline
\end{tabular}

Mean and maximum scan doses were calculated from the value of scan profile curve within image scan length.
The mean and SD dose with tube current modulation was $38.63 \pm 2.09 \mathrm{mGy}$ for the left lung- and $46.02 \pm 2.67 \mathrm{mGy}$ for the left breast region, i.e. 0.56 and 0.55 times lower than the mean dose without modulation (Table 2b). A comparison with the prospective scan mode indicated that the mean dose with modulation was 1.79 and 1.95 times higher in the left lung and breast.

Table 3 shows the radiation dose ratio between the overrange- and exposed scan dose (the radiation dose including the overrange and the image scan length). In the retrospective mode, the radiation dose ratio for the overrange scan dose ranged from 16 to $24 \%$ compared to $4 \%$ with the prospective mode.

\section{Image noise}

In retrospective mode, image noise was 12.6- and $12.3 \mathrm{HU}$ with and without the ECG-based tube current modulation technique, respectively; these values were $11.9 \mathrm{HU}$ in prospective mode.

\section{Discussion}

OSL dosimeters were used to determine the dose profiles for phantom left lung- and left breast regions corresponding

Table $2 b$ Mean and maximum scan doses with retrospective CTCA and ECG-based tube current modulation technique.

\begin{tabular}{lll}
\hline & Left lung & Left breast \\
\hline Mean scan dose (mGy) & $38.63 \pm 2.09$ & $46.02 \pm 2.67$ \\
Maximum scan dose (mGy) & 82.36 & 93.64 \\
Accuracy (\%) & 5.41 & 5.78 \\
CTDIvol (mGy) & 52.9 & \\
DLP (mGy cm) & 517.29 & \\
\hline
\end{tabular}

Mean and maximum scan doses were calculated from the value of scan profile curve within image scan length. 
Table 3 Radiation dose ratio between overrange radiation dose and exposed scan dose at prospective and retrospective CTCA.

\begin{tabular}{llll}
\hline & & \multicolumn{2}{l}{ Radiation dose ratio (\%) } \\
\cline { 3 - 4 } & & Left lung & Left breast \\
\hline Retrospective mode & Without modulation & 15.77 & 19.61 \\
Prospective mode & With modulation & 23.54 & 20.65 \\
\hline
\end{tabular}

Radiation dose ratio calculated as (overrange radiation dose)/(radiation dose within exposed scan length).

Exposed scan length $=$ overrange scan length + image scan length.

to the lung and breast regions in humans. As the OSL dosimeter allows the delivery of radiation doses at $0.05 \mathrm{~mm}$ intervals, the OSL dosimeter is quite useful for assessing the actual radiation dose along the z-axis.

The results obtained with OSL dosimeters provide the mean and maximum dose for overlapping regions in prospective mode. Prospective CTCA acquisition technique facilitates coverage of the whole heart in 3 or 4 steps. In a manner different from retrospective scanning, the radiation dose is delivered at predefined points of the cardiac cycle rather than during the entire cycle [12,13]. In our study, the maximum dose to the left lung- and left breast regions were $30.24 \%$ and $50.57 \%$ higher than the mean radiation dose. In retrospective mode, the dose profiles with ECG-based tube current modulation were significantly different from those without it; the mean radiation dose was lower by $55 \%$ with- than without ECG-based tube current modulation. An OSL dosimeter is also useful for determining an unnecessary dose outside the target area in retrospective mode using helical scanning. When the retrospective scan mode is applied, any unnecessary dose outside the target area is a critical issue. As the reconstruction algorithm requires additional raw data on both sides of the planned scan, extra rotations outside the planned length are needed for image reconstruction using helical scans [16]. In retrospective scanning mode, the unnecessary dose was calculated from the dose profile and ranged from 16 to 24\%; it increased with increasing beam width and beam pitch [17]. Because the overexposed length is expressed as the extra distance by the table feeds plus the beam width. When we examine our patients by CTCA, CTDIw or CTDIvol data are displayed on the CT console. However, these values do not reflect patient specifics such as the body size, and the region examined. In fact, our results for the mean and maximum doses are different from the CTDI values. In addition, measurement values derived from a point dose may not reflect the whole irradiation dose delivered to the region within the image scan length because the dose profile often changes along the $z$-axis when the dose modulation- and other dose reduction techniques are applied. Therefore, we suggest that the dose profile should be used to evaluate detailed information on the dose delivered to patients. This study has certain limitations. First, for measurements in the retrospective scan mode using helical scans, we aligned 2 OSL dosimeters along the $\mathrm{z}$-axis to evaluate the radiation dose outside the length of the image scan. Although the dose profiles did not affect the results, there may have been a slight decrease in the radiation dose between the ending edge of the first- and the top edge of the second OSL dosimeter. Second, we recorded the radiation dose at a heart rate of $60 \mathrm{bpm}$ using the ECG-based tube current modulation technique with the retrospective scanning mode. In clinical practice, the radiation dose reduction depends on the heart rate when the ECG-based tube current modulation technique is applied, because the peak effective current-time varies with changes in the $R-R$ interval. Finally, we returned the OSL dosimeters to the manufacturer for reading. Although we cannot rule out the possibility of fading during dosimeter handling and transport, we think the loss is minimal. In a preliminary study we compared the radiation dose measured at the phantom center position by an OSL dosimeter and a calibrated CT pencil ionization chamber. The CTDIc measurement values were $7.65 \mathrm{mGy}$ for the dosimeter $7.29 \mathrm{mGy}$ for the ionization chamber, respectively. According to Benevides et al. [19]. who studied fading on OSL dosimeters, light-induced fading was of greater concern than was loss of the measured dose due to fading. We ship the OSL dosimeters in holders that protect them from exposure to light to avoid a loss in the dose.

In conclusion, the OSL dosimeter for CT dosimetry is useful to measure not only the actual radiation dose but also the dose profiles along the z-axis. With the OSL dosimeter it is possible to obtain detailed information including data on the overlap- and overrange area in lung and breast regions at both prospective and retrospective CTCA.

\section{References}

[1] Leschka S, Scheffel H, Husmann L, Gamperli O, Marincek B, Kaufmann PA, et al. Effect of decrease in heart rate variability on the diagnostic accuracy of 64-MDCT coronary angiography. AJR Am J Roentgenol 2008;190:1583-90.

[2] Miller JM, Rochitte CE, Dewey M, Arbab-Zadeh A, Niinuma H, Gottlieb I, et al. Diagnostic performance of coronary angiography by 64-row CT. N Engl J Med 2008;359:2324-36.

[3] Oncel D, Oncel G, Tastan A, Tamci B. Evaluation of coronary stent patency and in-stent restenosis with dual-source CT coronary angiography without heart rate control. AJR Am J Roentgenol 2008;191:56-63.

[4] Mollet NR, Cademartiri F, van Mieghem CA, Runza G, McFadden EP, Baks T, et al. High-resolution spiral computed tomography coronary angiography in patients referred for diagnostic conventional coronary angiography. Circulation 2005;112:2318-23.

[5] Leschka S, Alkadhi H, Plass A, Desbiolles L, Grunenfelder J, Marincek $\mathrm{B}$, et al. Accuracy of MSCT coronary angiography 
with 64-slice technology: first experience. Eur Heart J 2005; 26:1482-7.

[6] Bastarrika G, Thilo C, Headden GF, Zwerner PL, Costello P, Schoepf UJ. Cardiac CT in the assessment of acute chest pain in the emergency department. AJR Am J Roentgenol 2009;193: 397-409.

[7] Alkadhi H. Radiation dose of cardiac CT-what is the evidence? Eur Radiol 2009;19:1311-5.

[8] Ketelsen D, Luetkhoff MH, Thomas C, Werner M, Buchgeister M, Tsiflikas I, et al. Estimation of the radiation exposure of a chest pain protocol with ECG-gating in dual-source computed tomography. Eur Radiol 2009;19:37-41.

[9] Hirai N, Horiguchi J, Fujioka C, Kiguchi M, Yamamoto $\mathrm{H}$, Matsuura N, et al. Prospective versus retrospective ECG-gated 64-detector coronary CT angiography: assessment of image quality, stenosis, and radiation dose. Radiology 2008;248: 424-30.

[10] Husmann L, Valenta I, Gaemperli O, Adda O, Treyer V, Wyss CA, et al. Feasibility of low-dose coronary CT angiography: first experience with prospective ECG-gating. Eur Heart J 2008;29:191-7.

[11] Hsieh J, Londt J, Vass M, Li J, Tang X, Okerlund D. Step-andshoot data acquisition and reconstruction for cardiac $x$-ray computed tomography. Med Phys 2006;33:4236-48.

[12] Scheffel H, Alkadhi $H$, Leschka S, Plass A, Desbiolles L, Guber I, et al. Low-dose CT coronary angiography in the step- and-shoot mode: diagnostic performance. Heart 2008;94: 1132-7.

[13] Klass $O$, Jeltsch $M$, Feuerlein S, Brunner $H$, Nagel HD, Walker MJ, et al. Prospectively gated axial CT coronary angiography: preliminary experiences with a novel lowdose technique. Eur Radiol 2009;19:829-36.

[14] Bauhs JA, Vrieze TJ, Primak AN, Bruesewitz MR, McCollough $\mathrm{CH}$. CT dosimetry: comparison of measurement techniques and devices. Radiographics 2008;28:245-53.

[15] Yukihara EG, Ruan C, Gasparian PB, Clouse WJ, Kalavagunta C, Ahmad S. An optically stimulated luminescence system to measure dose profiles in $\mathrm{x}$-ray computed tomography. Phys Med Biol 2009;54:6337-52.

[16] van der Molen AJ, Geleijns J. Overranging in multisection CT: quantification and relative contribution to dose-comparison of four 16-section CT scanners. Radiology 2007;242:208-16.

[17] Tzedakis A, Damilakis J, Perisinakis K, Stratakis J, Gourtsoyiannis N. The effect of $z$ overscanning on patient effective dose from multidetector helical computed tomography examinations. Med Phys 2005;32:1621-9.

[18] McNitt-Gray MF. AAPM/RSNA physics tutorial for residents: topics in CT. Radiation dose in CT. Radiographics 2002;22: 1541-53.

[19] Benevides L, Romanyukha A, Hull F, Duffy M, Voss S, Moscovitch M. Light induced fading in the OSL response of $\mathrm{Al}_{2} \mathrm{O}_{3}: C$. Radiat Meas 45: 523-526. 